\title{
A study of surface swelling caused by graphitisation during pulsed laser ablation of carbon allotrope with high content of $\mathrm{sp}^{3}$ bounds.
}

\author{
G.B.J. Cadot ${ }^{\mathrm{a}}$, J. Billingham ${ }^{\mathrm{b}, *}$, D.A. Axinte ${ }^{\mathrm{a}}$ \\ ${ }^{a}$ Machining and Condition Monitoring Group, Faculty of Engineering, University of Nottingham, NG7 2RD, UK \\ ${ }^{b}$ School of Mathematical Sciences, University of Nottingham, NG7 2RD, UK
}

\begin{abstract}
Experiments and theory are employed to investigate the laser ablation of boron doped diamond and tetrahedral amorphous carbon using nanosecond pulses. For a single pulse at low values of fluence, the laser induces a swelling of the surface due to graphitisation, whilst a high level of fluence leads to recession of the surface due to vaporization.

To understand and investigate the underlying phenomena during the diamond-laser interaction, a model has been developed to reliably and quickly predict the behaviour of the surface and the thickness of the heat affected zone. The model is based on conservation of heat and mass during the laser-workpiece interaction. It consists of a one-dimensional system of non-linear equations that models the material heating, evaporation, graphitisation and plasma shielding. There is excellent agreement between numerical and experimental results for the position of the interfaces up to a high laser fluence. This model is the first to investigate the ablation of diamond that is able to capture surface swelling due to the graphitisation of the diamond layer, the graphite thickness and the amount of ablated material within a single framework. Furthermore, the model provides a novel methodology to investigate the thermal stability of diamond-like carbon films. The activation energy for tetrahedral amorphous carbon is obtained using the model with an accuracy of $3.15_{-0.22}^{+1.0} \mathrm{eV}$.
\end{abstract}

\section{Introduction}

With its high capability to generate small details, pulsed laser ablation (PLA) offers new possibilities for microprocessing/texturing of a large variety of difficult-to-cut materials such as high strength $\mathrm{Ti} / \mathrm{Ni}$ based superalloys (e.g. Ti6Al4V, Inconel 718), ceramics (e.g. SiC, Al2O3) and super-hard materials (diamond, cubic boron nitride).

The current development of techniques to synthesise diamond (e.g. Chemical Vapour Deposition) drives the development of new technologies in diverse industrial applications. Diamond and related materials, such as tetrahedral amorphous carbon (ta-C) and polycrystalline di-

\footnotetext{
* Corresponding author

Email address: john.billingham@nottingham.ac.uk

(J. Billingham )
}

amond, offer remarkable properties compared to conventional materials: high diffusivity[8], extreme hardness, outstanding wear resistance, low friction [9] and low light absorption coefficients [10]. This unique set of properties, leads to numerous potential applications for diamond and related materials, such as wear resistant coating [11], cutting tools for ceramic materials in oil and gas exploration [12], two-dimensional pixel and strip detectors for UV and X-ray sources [13, 14], charged particle detection systems for high-energy physics experiments [15], heat sinks for power electronics [16], and optical windows [17].

In most advanced applications, "as-synthesised" bulk diamond requires post-processing to generate 3D (micro) features that add specific functionalities to the product. However, the generation of $2 \frac{1}{2} \mathrm{D}$ or $3 \mathrm{D}$ functional features in diamond structures by mechanical methods (e.g. grind- 
ing) is difficult due to the limited access of the cutting tools to minute part geometries while generally not being regarded as economically efficient. With this in mind, a material removal method that is not based on mechanical interaction between the tool and the workpiece would be much more appropriate for the shaping of diamond and related materials at a high level of geometrical detail while resulting in less mechanical damage to the diamond based structure [18]. The use of lasers for diamond through cutting is well documented [19] but recently Pulsed Laser Ablation (PLA), i.e. controlled depth material removal, has been recognized as one of the most efficient and suitable methods for the 3D shaping of diamond [20] with unique capabilities to generate micro-features/textures to support the development of advanced products made of this unique material. It has been reported that various laser sources (micro-, nano-, pico- and femtosecond) produce different levels of thermal damage of diamond structures during PLA [21]. Although microsecond PLA results in a good material removal rate [22], it produces less well-defined ablated features and secondary effects (e.g. thermal damage, cracks); the shorter pulses (nano [23, 24], pico [25] to femto [26] second) lead to reduced material removal rates but have greater accuracy and minimise heat-related side effects.

Considering the above, it seems that for industrial applications, machining with nanosecond lasers offers a good compromise between the removal rate and the quality of the detail achieved through ablation [27]. In most cases, the generation of features using PLA is done based on experimental knowledge of the material removal, i.e. beam footprint against a particular target material. Some attempts at using geometrical modelling (convolution of a known footprint along the laser beam path) of PLA to predict the surface micro-geometry have been reported [28]; although these can predict the ablated surface and incorporate various laser characteristics (e.g. power, repetition rate), these geometrical models suffer from not taking into account the physical phenomena that lead to material removal [29]. Therefore, they are not suitable for understanding thermal damage or possible phase change during the laser-workpiece interaction that is critical to assess the possible uses of processed diamond.

The prediction of defects and subsurface damage in diamond and related material targets requires a deep understanding of the physical phenomena that occur during pulsed laser ablation. Thus, diamond thermal damage has been investigated for a wide range of laser machining conditions [30, 31]. Time consuming and difficult studies show that the PLA of diamond and related materials is accompanied by a transition of the diamond to the thermodynamically stable phase, graphite. It is in good agreement with previous studies on annealing of diamond in furnaces showing similar graphitisation [32]. In the annealing studies, the speed of the phase transition is proportional to the furnace temperature $[2,3]$; this demonstrates the prevalence of the thermally driven phase transition of diamond. Thermal phase transition has also been highlighted as a characteristic of pulsed laser ablation in tetrahedral amorphous carbon (ta-C) [25], a form of incomplete diamond for which the amount of carbon-carbon bound sp3 (diamond like bound) is between $70 \%$ and $90 \%$, and CVD diamond [33, p. 394]. In effect, these studies have shown that the thickness of the graphite layer as a function of the temporal length of the pulse follows the same trend as the characteristic length of the heat penetration. Thus, thermally activated phase transition of diamond is one of the important mechanisms for the growth of a graphite layer near the surface during pulsed laser ablation. Although experimental investigations help to understand the main driver of diamond graphitisation, there are few studies investigating the thickness of this layer as a function of the pulse fluence. Furthermore, the kinetics of the growth of the graphite layer (as material swelling) and the ablation (as material removal) of diamond structure have never been studied. The graphite layer formed in the initial stages of the abla- 
tion process plays a dominant role in the laser absorption due to its high absorption coefficient compared to diamond and related material [33]. In the study by Kononenko et al. [25], a model is presented that calculates the maximum extent of the graphitic phase. Although it provides satisfactory predictions, the model assumes that the temperature of graphitisation is the quasi-stationary annealing threshold. This contradicts earlier experiments on diamond annealing which show that graphitisation is slow at the quasi-stationary annealing temperature and does not occur during short heating [2]. Furthermore, the model does not consider the kinematics of the position of the phase change, and the transition between the two phases is not taken into account until the end of the simulation. In effect, the positions of the interfaces (graphite/diamond and graphite/vacuum) are calculated after the end of the simulation using the maximum penetration of the quasistationary annealing temperature. Thus, this framework implicitly assumes that the graphite and diamond have the same optical and thermal characteristics.

To address these research gaps, a new model is developed to study the kinematics of the graphitisation of diamond during nanosecond PLA. The proposed model and investigation methods aim at providing better insight into the kinematics of the graphite layer formation in the diamond structure thereby predicting the thickness of the graphite layer as a function of the fluence. The model is one-dimensional, and consists of the conservation of heat and mass during the interaction; radial heat diffusion can be neglected because the thermal penetration depth is at least one order of magnitude smaller than the diameter of the laser spot. This provides a flexible framework within which to study the influence of the optical and thermal properties of the diamond on graphitisation. The dynamic positions of the interfaces (graphite/diamond and graphite/vacuum) are simulated, but cannot be measured experimentally, so the final position of the surface (graphite /vacuum) is used to validate the model for two types of di- amond: boron-doped diamond and tetrahedral amorphous carbon.

\section{Model for the graphitisation of diamond and re-} lated material during nanosecond pulsed laser ablation

The ablation process for diamond and related material can be separated into three phases:

1. Diamond heating: the laser interacts with the pristine diamond phase. The heat distribution is governed by the thermal diffusivity and the laser penetration depth inside the diamond [34]. The saturated vapour pressure is negligible due to the low surface temperature, so vaporisation does not occur during the diamond heating. This phase ends when the total thickness of graphite calculated using the Arrhenuis law at the surface of the diamond is equal to two layers of graphene $(\sim 0.7 \mathrm{~nm})$. The surface temperature is used to calculate the graphitisation rate of the diamond.

2. Graphitisation and ablation: the total thickness of the layer of graphite reaches 2 layers of graphene and the laser interacts with the graphite and diamond layer. A thin-layer (a few Ångström) of diamond is numerically transformed into graphite at the surface of the diamond [1]. At first, this layer does not have a strong influence on the temperature distribution as its thickness is too small to absorb a significant part of the laser energy. However, the graphite layer quickly grows and, due to its high absorption coefficient, the heat distribution is dramatically changed. The dynamic position of the interfaces is critical for the determination of the heat distribution during this phase. A new modelling framework has therefore been used to correctly describe it, which represents the key element of this paper and is described in this section. 
3. Relaxation of the system: graphitisation stalls due to a lack of heat propagation inside the target. However, the surface temperature can still be above the ablation threshold resulting in the vaporisation of a part of the graphite layer. The new modelling framework can also be used to simulate this phase.

The model for the diamond heating phase has already been described elsewhere [34]. The physical phenomena are modelled to improve understanding of the ablation process during the graphitisation and ablation phase. Nanosecond laser ablation, that is to be considered in this paper, is a thermal process that is induced by the absorption of the laser pulse by the electrons in the target followed by a rapid $(<10 \mathrm{ps}$ ) thermalisation between the electrons and the lattice. The resulting heat then diffuses within the target. The thermal penetration depth, defined as $\mathrm{d}_{\text {Thermal }}$ $=\sqrt{2 D \tau_{\text {pulse }}}$ (where $D$ is the diffusivity and $\tau_{\text {pulse }}$ is the temporal length of the laser pulse), is at least one order of magnitude smaller than the diameter of the laser spot on the target. Thus, the heat distribution within the target workpiece can be determined using a 1D model. In this approach, the two layer (graphite and diamond) system is modelled (based on mass and energy conservation) to take into account graphitisation during the irradiation. In the following equations the subscripts $d$ and $g$ are used for the thermophysical and optical properties of diamond and graphite respectively. The vacuum/graphite and graphite/diamond interfaces are at $x=x_{1}$ and $x=x_{2}$ respectively.

The density difference between graphite and diamond leads to an outward displacement of the graphite, or swelling, of the graphitised surface as shown in Figure 1. Mass conservation at the diamond-graphite interface leads to

$$
v_{\text {swelling }}=\frac{\rho_{g}-\rho_{d}}{\rho_{g}} \partial_{t} x_{2}
$$

where $\rho$ is the density of the material and $v_{\text {swelling }}$ is the speed induced in the graphite due to the graphitisation.
The heat equation for the graphite is, using (1),

$$
\partial_{t} H_{g}=\partial_{x}\left[v_{\text {swelling }} H_{g}+\mathrm{D}_{g} \partial_{x} H_{g}\right]+S_{g}
$$

with $H, D$ and $S$ being enthalpy of the material, thermal diffusivity and the heat source induced by the laser respectively. The enthalpy is generically calculated as

$$
H(T)=\int_{T_{0}}^{T} \rho(T) c_{p}(T) d T
$$

with $c_{p}$ and $T$ being the specific heat and temperature respectively. The source term $S$ from the laser beam is

$$
S_{g}=\left(1-R\left(T_{\text {surface }}\right)\right) \alpha_{g} \exp \left[-\alpha_{g}\left(x-x_{1}\right)\right] i(t),
$$

with $R, \alpha, T_{\text {surface }}$ and $i(t)$ being reflectivity, absorption coefficient for the laser wavelength, surface temperature, and the temporal profile of the laser pulse respectively. It has been shown that the absorption of a significant fraction of the laser energy by the plasma that is created above the surface is an important phenomenon during nanosecond laser ablation [34], [35]. Including plasma absorption can lead to significant improvement of model predictions [36, $37,38,39]$. In this context, using the method developed for graphite ablation by Bulgakova et al. [40], the plasma absorption is estimated using the total optical thickness of the ablation plume $\Lambda$ such that

$$
\Lambda(t)=a x_{a}(t)+b E_{a}(t)
$$

with $E_{a}(t)$ and $x_{a}(t)$ being the energy in the plasma and the depth ablated respectively. Here $a$ and $b$ are free parameters usually determined by fitting the model to experimental data. This simple model for the optical thickness is based on the hypothesis than an increase in the plasma energy, $E_{a}(t)$, or the plasma density increases the absorption. Thus, the plasma shielded heat source is

$\left.S_{g}=\left(1-R\left(T_{\text {surface }}\right)\right) \alpha_{g} \exp \left[-\alpha_{g}\left(x-x_{1}\right)\right)\right] \exp [-\Lambda(t)] i(t)$

The heat equation for the diamond is

$$
\partial_{t} H_{d}=\partial_{x}\left[\mathrm{D}_{d} \partial_{x} H_{d}\right]+S_{d}
$$




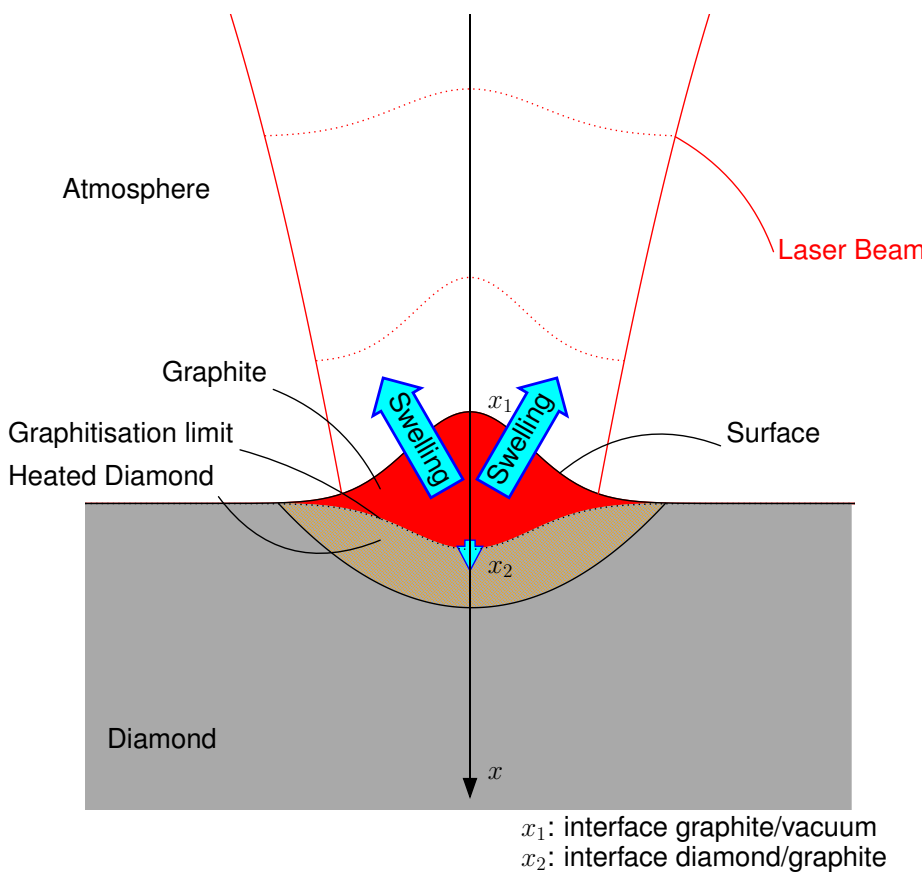

(a)

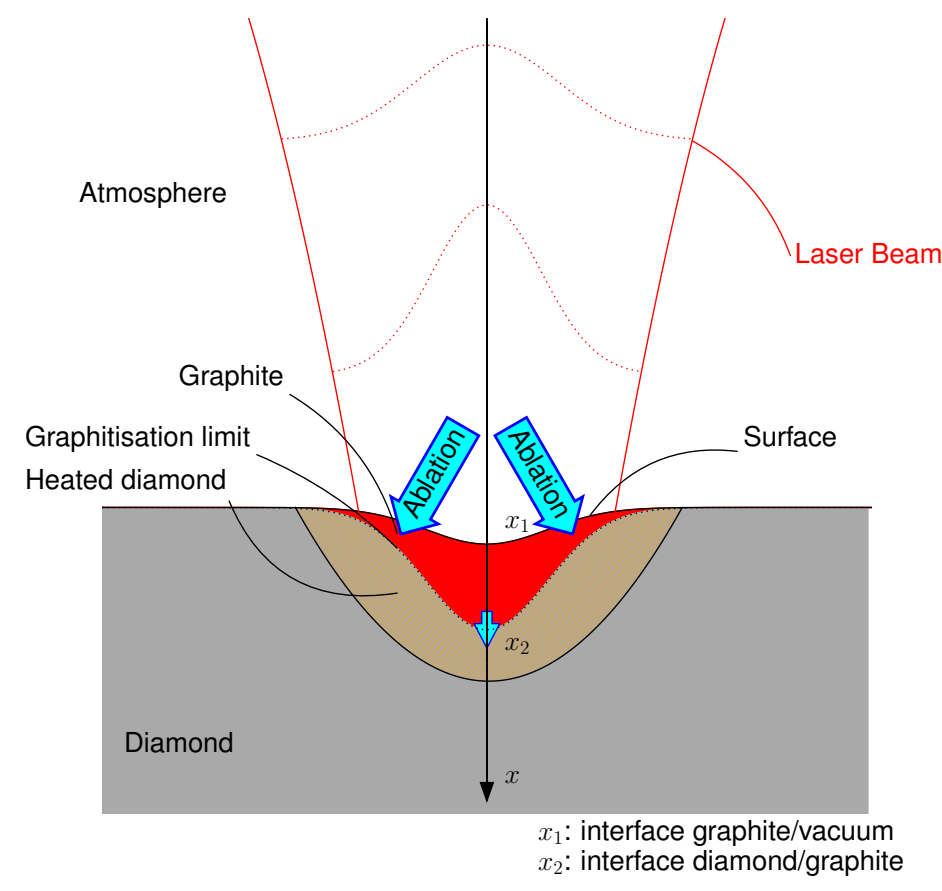

(b)

Figure 1: a) Scheme of diamond ablation at low fluences. b) Scheme of diamond ablation for high fluences.

where the source term $S_{d}$ is

$$
\begin{aligned}
S_{d}= & \left(1-R\left(T_{\text {surface }}\right)\right) \alpha_{d} \exp \left[-\alpha_{d}\left(x-x_{2}\right)-\alpha_{g}\left(x_{2}-x_{1}\right)\right] \\
& \exp [-\Lambda(t)] i(t) .
\end{aligned}
$$

The system is completed by the Stefan condition,

$$
\left.\mathrm{D}_{g} \partial_{x} H_{g}\right|_{x=x_{2}}-\left.\mathrm{D}_{d} \partial_{x} H_{d}\right|_{x=x_{2}}=\partial_{t} x_{2} \rho_{d} \Delta h_{d-g},
$$

with $\Delta h_{d-g}$ the heat of graphitisation and the jump of enthalpy at the graphite-diamond interface

$$
\left.h_{g}\right|_{x=x_{2}}-\left.h_{d}\right|_{x=x_{2}}=h_{g}\left(T_{\text {interface }}\right)-h_{d}\left(T_{\text {interface }}\right),
$$

where $T_{\text {interface }}$ is the temperature at the interface between diamond and graphite. Note that the Stefan condition has been modified to take into account the fact that graphitisation is irreversible and follows Arrhenuis law, (unlike melting or solidification phase changes) thus the speed of the interface between the graphite and the diamond cannot be negative and is calculated using the following equation,

$$
\partial_{t} x_{2}=C \exp \left[\frac{-\Delta E}{R T_{\text {interface }}}\right]
$$

with $C$ and $\Delta E$ being respectively a constant representing the rate of transformation between graphite and diamond, and the activation energy for the transition between diamond and graphite. The domain of solution is finite, and an adiabatic boundary condition is applied at the bottom, $x_{\max }$, so that

$$
\left.\left[D_{d} \partial_{x} H_{d}\right]\right|_{x=x_{\max }}=0
$$

We always choose $x_{\max }$ large enough that the temperature there does not change significantly during the simulation. At the top of the domain, the boundary condition is controlled by evaporation. Thus, the energy balance at the irradiated surface is

$$
\left.\mathrm{D}_{g} \partial_{x} H_{g}\right|_{x=x_{1}}=v_{a} \Delta H_{\mathrm{vap}},
$$

with $v_{a}$ and $\Delta H_{\text {vap }}$ being the speed of ablation and the enthalpy of vaporization [41] respectively. The relation 
between the rate of evaporation and vapour pressure is $[42]$

$$
m=\sqrt{\frac{M}{2 \pi R T_{s}}} P_{v}
$$

with $M, R, T_{s}$ and $P_{v}$ being molar weight, the gas constant, surface temperature and the saturated vapour pressure respectively. The saturated vapour pressure is estimated using the Clausius-Clapeyron equation,

$$
\frac{d P_{v}}{d T}=\frac{\Delta H_{v a p}}{T \Delta v}
$$

with $\Delta v$ the change in specific volume during the evaporation. The vapour is considered to behave like an ideal gas and the latent heat of vaporization is regarded as a constant over the range of temperatures encountered [43]. Since it has been shown that a certain proportion of the atoms from the vapour are reflected to the surface [44], a sticking coefficient $\beta$ is included in (16) to account for this phenomenon. Using (14) and (15), it is possible to obtain the ablation velocity of the graphite,

$$
v_{a}=(1-\beta) \sqrt{\frac{M}{2 \pi R T_{s}}} \frac{p_{0}}{\rho_{g}} \exp \left[\frac{\Delta H_{v a p}}{R}\left(\frac{1}{T_{0}}-\frac{1}{T_{s}}\right)\right],
$$

where $T_{0}$ is the reference temperature under the reference pressure $p_{0}$. The saturated pressure for carbon is tabulated for a range of temperatures [45] with reference pressure and temperature $p_{0}=100 \mathrm{kPa}$ and $T_{0}=3908 \mathrm{~K}$. The position of the interface between graphite and vacuum is determined by

$$
\partial_{t} x_{1}=v_{\text {swelling }}+v_{a}
$$

Finally, a front fixing method [46] is used to map the domain of solution to a field domain which is more convenient for numerical solution.

At the start of the simulation, the temperature of the diamond is set at $300 \mathrm{~K}$. Initially, the laser interacts directly with the diamond, so just the equation for the flow of heat in the diamond is solved. The surface temperature in the diamond increases until the total thickness of the graphite layer calculated using the Arrhenuis relation reaches twice the thickness of graphene layer. Once this thickness of the graphite layer is attained, the diamond is numerically transformed into graphite by instantaneously replacing a small layer of diamond (two layer of graphene thickness ) with graphite. Afterwards, the two heat equations are solved simultaneously and the interfaces (graphite/diamond and graphite/vacuum) evolve according to (17) and (9).

The system of equations is discretised using Taylor series and finite volume methods. Temporal discretisation is achieved using the implicit Euler method. The solver uses an adaptive time step dependent on the value of the ablation speed. A high ablation speed means a small time step. The matrix resulting from the discretisation is inverted using the library SUPERLU [47]. The solver is second order in space and first order in time. At each time step, the material properties are evaluated using the enthalpy from the previous time step. The simulation is carried out until the system is not subject to vaporisation and graphitisation, usually about $2 \mu \mathrm{s}$. The size of the simulated sample is around $2 \mu \mathrm{m}$ for tetrahedral amorphous carbon and 20 $\mu \mathrm{m}$ for boron doped diamond. The temperature of the sample at the end of the simulation typically is around 20 $\mathrm{K}$ over the initial value.

\section{Methodology for material properties used in the simulation}

In this study, the model has been tested against two types of diamond: boron doped diamond (BDD) and tetrahedral amorphous carbon (ta-C). These materials are chosen because they present dissimilar optical and thermal properties, as well as a notable difference in microstructure. BDD is a microcrystalline diamond exhibiting a high diffusivity and low absorption coefficient at the wavelength of the laser used in the experimental investigations (1061 $\mathrm{nm})$. In contrast, ta-C is an amorphous diamond like carbon having a low diffusivity and high absorption coefficient at the laser wavelength used during the experimental tests 
(248 nm) [33]. Previous studies show that the temperature dependence of the material properties (thermal and optical) has a strong influence on the predictions of models for nanosecond pulsed laser ablation [48]. Thus, it is critical for the accuracy of the model results to employ material properties as close as possible to the real ones. The methodology for the determination of the material properties (optical and thermal) for the two target materials and graphite will be presented below. The thermal properties used in the model are the specific heat capacity, the density, the thermal diffusivity, the specific enthalpy of graphitisation and the temperature of graphitisation for the diamond material. The optical properties used in the model are the reflectivity and the absorption coefficient.

\subsection{Optical and thermal properties for boron doped dia- mond (BDD)}

The optical $[53,54,55,56]$ and thermal $[57,58]$ properties of boron doped diamond have been widely reported in the literature. However, there is little data for the sample used in this study (a micro-crystalline diamond with a boron doping rate of $\sim 3 \times 10^{20} \mathrm{~cm}^{-3}$ ), due to the wide range of microstructural composition (single crystal, micro-crystalline and nano-crystalline) and doping rate $\left(10^{16}\right.$ to $10^{21} \mathrm{~cm}^{-3}$ ) available. Furthermore, previous studies have shown that boron doping rate influences the optical properties and some thermal properties. The thermal and optical properties used in the simulation are:

- Specific heat: previous studies show that the specific heat of boron doped diamond is not dependent on the boron doping rate [15][p. 476]. Furthermore, the specific heat of diamond does not depend on its microstructure (single-crystalline, micro-crystalline, nano-crystalline) [59]. Thus, the specific heat of single crystal diamond is used in the model for the specific heat of boron doped diamond [49].

- Thermal conductivity: the boron doping rate in diamond affects the thermal conductivity [60, 61] by up to one order of magnitude. This is due to the increase of point defects and boron concentration in the grain boundaries which increases the phonon dispersion. The thermal conductivity reported in the manufacturer data sheet[49] is used in the model. Although, the thermal conductivity should decrease with an increase of temperature, the reported data only includes the value at $300 \mathrm{~K}$. Thus, the thermal conductivity is considered as constant over the range of temperature studied.

- Density: the density of boron doped diamond is considered to be equal to the density of pure diamond [61].

- Specific enthalpy of graphitisation: the specific heat of graphitisation has been experimentally measured in the work of Rossini et al. [52].

- Activation energy and rate of graphitisation: the graphitisation rate as a function of temperature for pure diamond has been previously reported $[2,3]$.

- Reflectivity: the complex part of the refractive index is relatively small compared to its real part [56]. Thus, the real part of the refractive index alone is meaningful for the calculation of the refractive index. Experimental studies show that the refractive index has a negligible dependence on the boron doping rate [56]. Furthermore, the reflectivity of a microcrystalline boron doped diamond with a doping rate at $1.3 \times 10^{20} \mathrm{~cm}^{-3}$ has been reported in the literature [62] and is used in the model.

- Absorption coefficient: the absorption coefficient at $1061 \mathrm{~nm}$ (the laser wavelength used in the experimental tests) is highly dependent on the boron doping rate $[53,54]$, and is approximately $6 \times 10^{5} \mathrm{~m}^{-1}$ at $300 \mathrm{~K}$ for the BDD sample used in this study. Furthermore, Hall mobility and carrier concentration 
Density [49], g.cm ${ }^{-3}$

Absorption coefficient @1061 [50], m-1

Reflectivity@1061nm [51],

Specific enthalpy of graphitisation [52], J.g ${ }^{-1}$

Rate of graphitisation [3], m.s ${ }^{-1}$

Activation Energy [3], J.mol ${ }^{-1} / \mathrm{eV}$

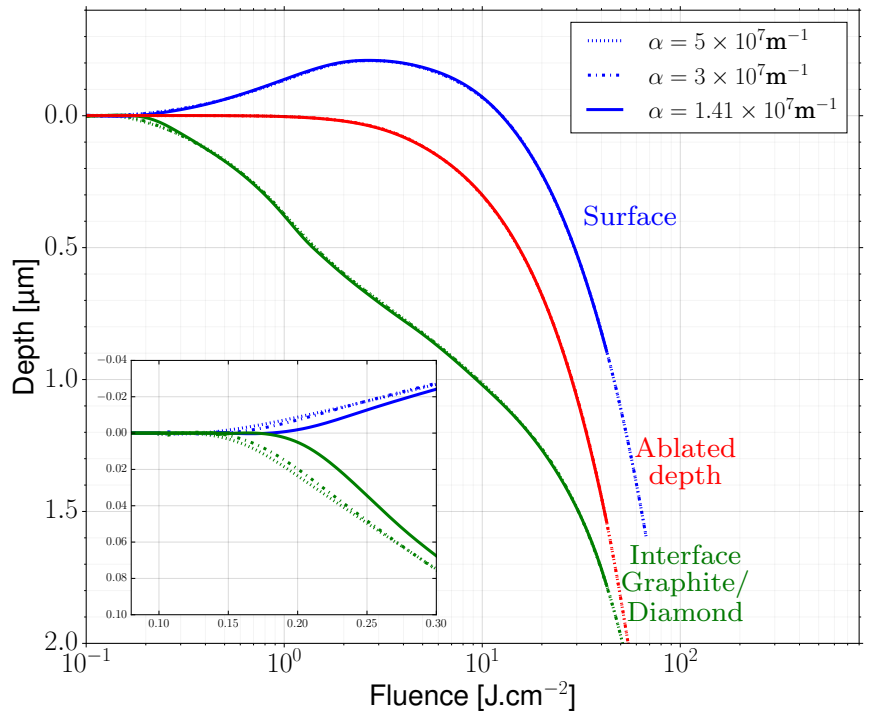

Figure 2: Position of the interfaces for ta-C with a constant reflectivity $(\mathrm{R}=0.45)$ for the graphite at $248 \mathrm{~nm}$ without considering the plasma absorption. The red, blue and green lines are respectively the ablated depth, the interface position of graphite/vacuum and interface position of diamond/graphite.

measurements show that the absorption coefficient should decrease with an increase of temperature [53]. The thermal and optical properties for the boron doped diamond used in the model are summarised in Table 1.

\subsection{Optical and thermal properties for tetrahedral amor- phous carbon}

The thermal and optical properties of tetrahedral amorphous carbon are not commonly reported in the literature since their measurement is difficult in the thin films that are typically deposited [68, p. 158-162]. However, it has been established that the fraction of $\mathrm{sp}^{3} / \mathrm{sp}^{2}$ carboncarbon bound is linked to the density [63], the thermal conductivity [67], the specific heat [66] and the optical properties [69]. The thermal and optical properties are usually measured at $300 \mathrm{~K}[64,33]$ :

- Specific heat: the work of Hakovirta et al. [66] suggests that the specific heat of diamond like carbon films can be calculated using the specific heat of the graphite and diamond, the ratio of $\mathrm{sp}^{3} / \mathrm{sp}^{2}$ and the hydrogen concentration in atomic percentage. Thus, the specific heat used for this study is

$$
c_{p_{\mathrm{taC}}}(T)=r_{s p^{3} / s p^{2}} c_{p_{\mathrm{d}}}(T)+\left(1-r_{s p^{3} / s p^{2}}\right) c_{p_{\mathrm{g}}}(T)
$$

with $r_{s p^{3} / s p^{2}}$ the ratio of sp3/sp2 bound, $c_{p_{\mathrm{d}}}(T)$ the specific heat of diamond and $c_{p_{\mathrm{g}}}(T)$ the specific heat of graphite.

- Thermal conductivity: a previous study demonstrates that the thermal conductivity is linearly dependent on the ratio of the $\mathrm{sp}^{3} / \mathrm{sp}^{2}$ bound and weakly dependent on the temperature around $300 \mathrm{~K}$ [67]. Thus, the thermal conductivity is considered as constant over the range of temperature used.

- Density: the film density is linearly dependent on the ratio of the $\mathrm{sp}^{3} / \mathrm{sp}^{2}$ bound [63].

- Specific enthalpy of graphitisation: since the change 
Density [63], g.cm ${ }^{-3}$

Ratio sp3/sp2 [64], \%

Absorption coefficient @248nm [65], m

Reflectivity @248nm [65]

Specific enthalpy of graphitisation [52], J.g ${ }^{-1}$

Rate of graphitisation, optimized see $11, \mathrm{~m} . \mathrm{s}^{-1}$

Activation Energy, optimized see 11, J.mol ${ }^{-1} / \mathrm{eV}$

Specific Heat [66],

of enthalpy between graphite and diamond is the energy released from the sp3 carbon-carbon bound, the specific enthalpy of graphitisation depends linearly on the ratio of $\mathrm{sp} 3 / \mathrm{sp} 2$ bound [52].

- Activation energy and rate of graphitisation: the activation energy for ta-C has been previously reported $[4,5]$ however due to the thickness of ta-C the most accurate value available is $3.5 \pm 0.9 \mathrm{eV}$. Furthermore, the rate of graphitisation has not been measured. Thus, the activation energy and the rate of graphitisation are free parameters that are determined by fitting the experimental results and the calculated data on the surface position. In this work, the model is validated for boron doped diamond, then the same model is applied for ta-C. It is then possible to obtain accurate values for the activation energy and rate of graphitisation for ta-C.

- Reflectivity: Tay et al. [69] have measured the optical properties of ta-C film for a ratio of $\mathrm{sp}^{3} / \mathrm{sp}^{2}$ bound around $80-85 \%$ and found a weak dependence of refractive index at $250 \mathrm{~nm}$ on the ratio of $\mathrm{sp}^{3} / \mathrm{sp}^{2}$ bound. The reflectivity measured by Larruquert et al. [65] is used in the model for the reflectivity of the ta-C film at $248 \mathrm{~nm}$.

- Absorption coefficient: the absorption coefficient at $248 \mathrm{~nm}$ for ta-C is highly dependent of the ratio of $\mathrm{sp}^{3} / \mathrm{sp}^{2}$ bound[69]. Numerical experiments has been performed showing that the model predictions are not noticeably influenced by the absorption coefficient of ta-C, see Figure 2. Thus, a good accuracy for the absorption coefficient is not necessary to provide accurate numerical results.

The thermal and optical properties for the ta- $\mathrm{C}$ used in the model are summarised in the Table 2.

\subsection{Optical and thermal properties for graphite}

The optical and thermal properties of graphite are commonly reported in the literature. In this study, the graphite is considered to be non-porous during the ablation process [30] and polycrystalline [76].

- Specific heat: the specific heat of graphite has been reported in numerous works for many varieties of graphite (pyrolytic, porous, etc.) [74, 77, 34]. The specific heat reported in the different studies are in fairly good agreement from $300 \mathrm{~K}$ to $4800 \mathrm{~K}$. Thus, the value of the specific heat used in the model for graphite is the one reported in [74]. For a temperature over $4800 \mathrm{~K}$, experimental measurements suggest that the specific heat of graphite is constant, [78].

- Thermal conductivity: the thermal conductivity of porous graphite (16\%) was reported in [34]. For non- 
Density [71], g.cm ${ }^{-3}$

Temperature of reference [45], K

Latent heat of vaporization [34], $\mathrm{kJ} \mathrm{mol}{ }^{-1}$

Absorption coefficient [72] @1061, m

Absorption coefficient [72] @248, m-1

$1.12 \times 10^{8}$

Reflection coefficient [34] @1061nm

$0.21-2.83 \times 10^{-5}(T-300)$ for $T \leq 7000 \mathrm{~K}$

0.02 for $T>7000 \mathrm{~K}$

Reflection coefficient [72, 73] @248nm

$0.45-5.22 \times 10^{-5}(T-300)$ for $T \leq 7000 \mathrm{~K}$

0.10 for $T>7000 \mathrm{~K}$

Specific Heat, [74]

See Figure 3a)

Thermal diffusivity, [34, 75]

See Figure 3b)

porous material, the thermal conductivity is calculated using the Maxwell Garnett model [79]. The thermal conductivity used in the model for graphite is $25 \%$ higher than thermal conductivity reported by Bulgakova et al. [34].

- Density: the density of non-porous graphite has been reported to be $2.2 \mathrm{gcm}^{-3}$, [71].

- Reflectivity at $1061 \mathrm{~nm}$ : the reflectivity of graphite at $1061 \mathrm{~nm}$ has been previously reported [34], see Table 3 .

- Reflectivity at $248 \mathrm{~nm}$ : the reflectivity of graphite at $248 \mathrm{~nm}$ has been measured at $300 \mathrm{~K}$ [72]. Furthermore, pump and probe experiments show that the reflectivity of graphite for three different wavelengths decreases with temperature [73]. It is likely that the reflectivity at $248 \mathrm{~nm}$ follows the same trend, so it is assumed that the reflectivity decreases with temperature down to 0.10 at $7000 \mathrm{~K}$. It is believed that a reduction of the reflectivity with temperature represents a closer approximation to reality than a constant reflectivity. The value of the reflectivity taken for graphite at $248 \mathrm{~nm}$ is given in Table 3. Further- more, numerical experiments are presented in the Subsection 5.5.2 to understand the relationship between the reflectivity and the position of the interfaces (graphite/vacuum and graphite/diamond).

- Absorption coefficient at 248 nm and 1061 nm: Djurišić et al. have measured the absorption coefficient of graphite at $248 \mathrm{~nm}$ and $1061 \mathrm{~nm}$ at $300 \mathrm{~K}$ [72]. It must be noted that pump and probe experiments demonstrate a decrease of the absorption coefficient with temperature. For the sake of simplicity, the absorption coefficient is considered to be constant.

The optical and thermal properties used in the model for graphite are given in Table 3.

\section{Experimental Methodology}

The model developed in this study is used to simulate the phase transition between diamond and graphite under high thermal load. This effect is particularly important during the first interaction between the laser and the diamond target (single pulse ablation). In effect, the creation of a graphitic layer over the diamond leads to a large variation of material properties, which dramatically changes 




(a)

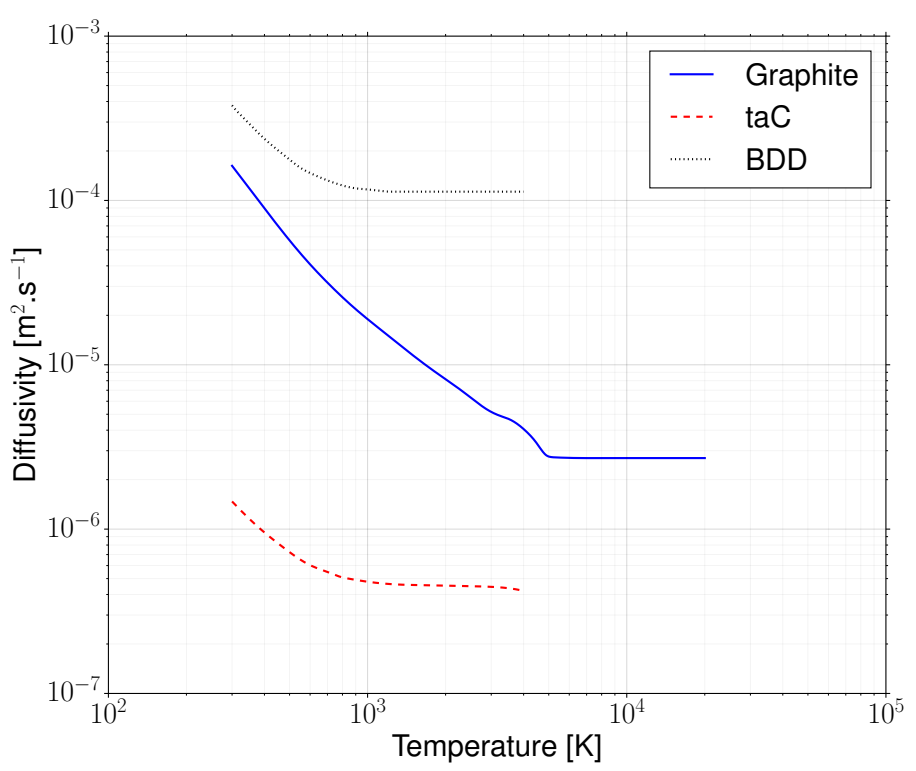

(b)

Figure 3: Material properties as a function of the temperature for the three materials used in the model for (a) the specific heat and (b) the diffusivity.

the heat distribution inside the target. Subsequent interactions between the laser and the diamond target (multipulse ablation) do not create such a dramatic change in the heat distribution since a graphite layer is already present on top of the diamond. Single pulse ablation tests are the most stringent, and are therefore used to investigate the capabilities of the model for the two dissimilar diamond and related materials.

The methodology and experimental results for tetrahedral amorphous carbon have been reported previously $[80,33]$. Therefore, the experimental setup is briefly described in the following paragraph and the reader is referred to the previously published studies for further information. However, the results for boron doped diamond have not been previously published, and the experimental methodology used in this study is detailed below.

\subsection{Tetrahedral amorphous carbon (ta-C)}

A KrF eximer laser producing 20 ns (FWHM) pulses at $248 \mathrm{~nm}$ is used to study the ablation of $2 \mu \mathrm{m}$ thick taC. The height and depth of the crater is measured for a range of fluence between 0.1 and $600 \mathrm{~J} . c m^{-2}$. The $2 \mu \mathrm{m}$
ta-C film is produced by pulsed arc deposition, using a technique described in [64].

\subsection{Boron doped diamond (BDD)}

The experimental tests for the model validation are conducted with a fiber laser at constant pulse repetition rate of $35 \mathrm{kHz}$. The temporal profile is characterised by a full-width at half-maximum of $30 \mathrm{~ns}$ with a long trail after the maximum that lasts approximately 200 ns. The exact temporal profile of the laser pulse is obtained from the manufacturer data sheet and an example is provided in Figure 4. Laser pulses are fed into a galvanometers head mounted with a $100 \mathrm{~mm}$ focal length $\mathrm{f}-\theta$. The resulting spatial profile is Gaussian with a diameter around $38 \mu \mathrm{m}$ (using the $1 / \mathrm{e}^{2}$ definition). The maximum power achievable at the focal plane is $18.48 \mathrm{~W}$ and the maximum energy per pulse is $0.53 \mathrm{~mJ}$. Thus, it is possible to obtain fluences

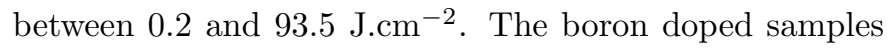
are produced by Element Six Ltd. The samples are polished on both sides $(\mathrm{Ra}<30 \mathrm{~nm})$ and have a thickness around $0.5 \mathrm{~mm}$. The beam moves on the target surface with a high speed $\left(>4000 \mathrm{~ms}^{-1}\right)$ so that two consecutive 
pulses do not overlap and at least ten craters are produced and averaged in order to reduce the error arising from variability of the laser parameters. Each crater is measured using a white light interferometer, Bruker GT-i, that provides accurate measurement (error $<10 \mathrm{~nm}$ ) of the surface after the ablation. Afterwards, the radial profile and depth at the centre of the crater is extracted, see Figure 9.

\section{Results and discussions}

The model is tested for two dissimilar diamond and related materials, a tetrahedral amorphous carbon and a boron doped diamond. For tetrahedral amorphous carbon, the influence of the graphite reflectivity on the position of the interfaces is discussed. The numerical predictions are then compared to the experimental results. Finally, the discrepancies between predictions and experimental results are discussed. For boron doped diamond, the experimental results are presented and reviewed. Then, the influence of the absorption coefficient of boron doped diamond for the position of the interfaces is presented. Finally, the discrepancy between the numerical and experimental results is investigated.

\subsection{Boron doped diamond}

In the case of boron doped diamond at low fluence, the swelling is clearly visible in Figure 5(a). SEM imaging (Figure 5(a)) suggests that the swelling occurs following the preferential direction and that the microstructure has a small influence on the final topography. Previous investigation shows that a variation in the doping rate over the surface exists [81]. This creates local variability in the amount of absorbed energy during pulsed laser ablation, leading to variation in the amount of swelling. Thus, this explains the higher variability observed for the depth/height of the crater for low fluence. SEM imaging for high fluence does not show such variability in the shape of the crater, see Figure 5(b). For high fluence, the graphitisation occurs earlier, see Figure 4, thus a larger

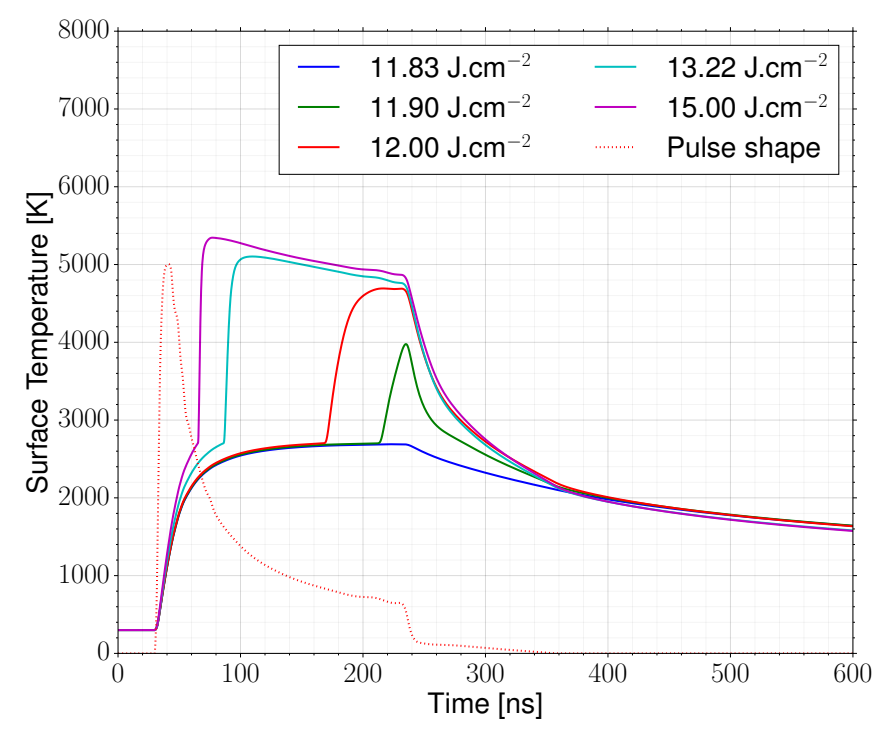

Figure 4: Surface temperature as a function of time for several fluence and the pulse shape for the SPI-G3.

part of the laser pulse interacts directly with the graphite for which boron doping rate does not affect the absorption coefficient of the graphite. This leads to a lower variation of ablation/swelling over the surface at high fluence.

As mentioned previously, the absorption coefficient of boron doped diamond has a strong influence on the results of the simulation. The influence of the absorption coefficient on the simulation cannot be estimated easily because the moment the graphitisation starts the properties of the surface material change dramatically, see Figure 4 . The absorption coefficient for square diamond is two orders of magnitude lower than the coefficient for graphite, which leads to a steep increase of temperature after the start of the graphitisation at the surface, see Figure 4. Therefore, numerical experiments have been performed to quantify the influence of the absorption coefficient on the simulation, see Figure 6.

The numerical results show a marked influence of the absorption coefficient on the position of the interfaces for the whole range of fluence studied, see Figure 6. First of all, the time at which graphitisation begins is highly dependent on the absorption coefficient of square diamond, and the final position of the interfaces is highly dependent 




(a)

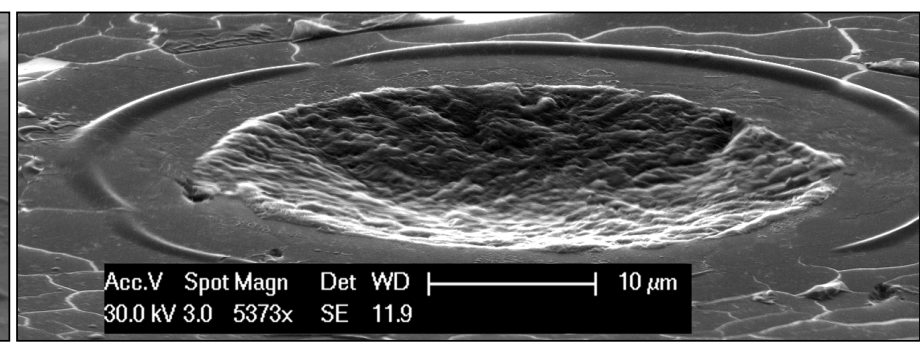

(b)

Figure 5: SEM imaging of crater for a fluence of (a) $12.48 \mathrm{~J} . c m^{-2}$ and (b) $65.9 \mathrm{~J} . \mathrm{cm}^{-2}$ with an angle of $75^{\circ}$.

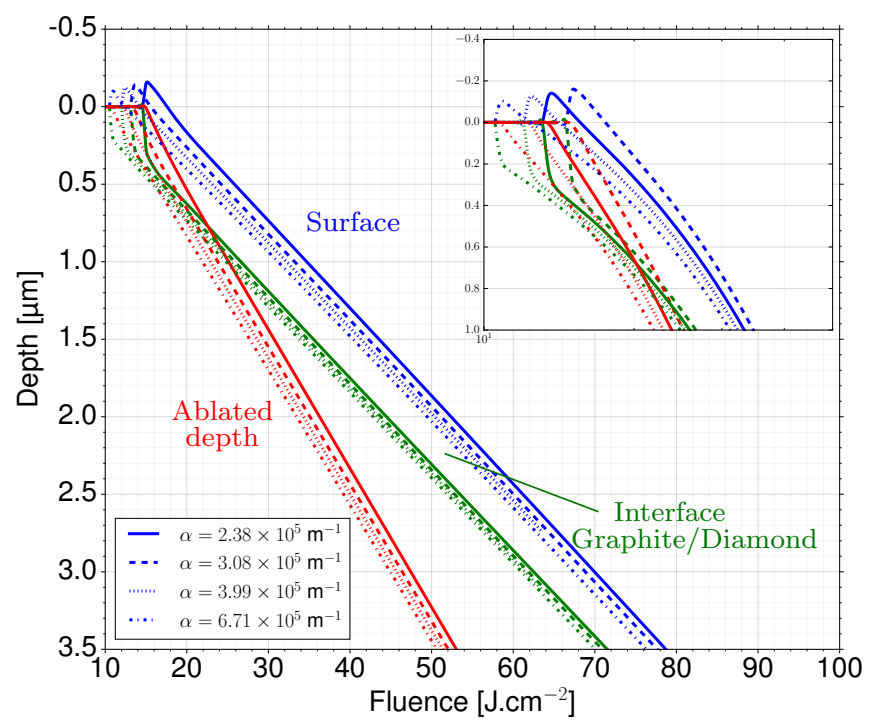

Figure 6: Interfaces position as a function of the fluence for 3 different values of the boron-doped diamond absorption coefficient. The blue, red and green line are the position of the graphite/vacuum interface, the depth of ablated graphite and the position of the graphite/diamond interface respectively.

on this time. The jump of absorption coefficient of the surface material leads to a dramatic change in the heat distribution, with a significant fraction absorbed in the first 42 $\mathrm{nm}$ of the graphite layer. The large amount of heat generated at the surface quickly diffuses inside the graphite, leading to a rapid swelling of the surface by tens or hundreds of nanometres. Furthermore, the diamond graphitisation is accompanied by a strong vaporisation at the surface of the graphite. Thus, the start of graphitisation will affect greatly the distribution of the heat. Interestingly, the position of the interface between the graphite and the diamond converges to one curve for high fluence, which suggests that the BDD absorption coefficient mostly affects the amount of energy used to evaporate the graphite.

In the results presented in Figure 7, the absorption coefficient is determined by fitting the experimental results and the calculated position of the interface graphite/vacuum. It has been found that an absorption coefficient for the boron doped diamond of $5.18 \times 10^{5} \mathrm{~m}^{-1}$ gives good results, see Figure 7. It is close to the value measured at $300 \mathrm{~K}$ (approximately $7 \times 10^{5} \mathrm{~m}^{-1},[53]$ ). Furthermore, it has been shown that the absorption coefficient should decrease with an increase of temperature[54], so the value found is consistent with the available experimental data. The experimental results show that the amount of ablated material increases linearly up to a fluence of $50 \mathrm{~J} . \mathrm{cm}^{-2}$, which suggests that the energy of the laser beam is totally coupled to the target and that the amount of energy absorbed by the plasma over the crater is small, see Figure 7. This is substantiated by the good agreement between the model without plasma absorption and the experimental results.

At higher powers, when plasma absorption is neglected the amount of ablated material is lower than the model prediction. This suggests that a part of the energy is shielded from the target by the plasma. However, when the method presented above was used to estimate the plasma absorption, (5), no values of the parameters $a$ and $b$ could be found to reproduce the experimental results. The failure of the plasma model to correctly predict the amount of energy absorbed is due to several factors. The main ab- 


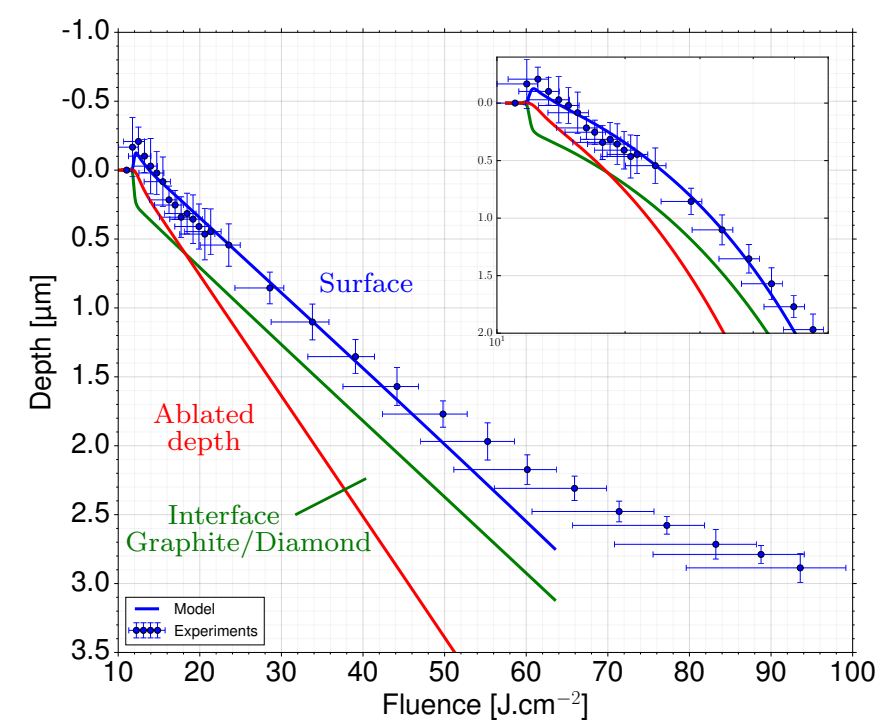

Figure 7: Position of the interfaces for the model and the experiments for BDD. The red, blue and green lines are the ablated depth, the interface position of graphite/vacuum and interface position of diamond/graphite respectively. The inset show a subset of the data in a logarithm scale for the fluence.

sorption mechanism during infrared pulsed laser ablation is via inverse Bremsstrahlung, which is highly dependent on the temperature and density of electrons [82]. Thus, the plasma absorption will present an ignition threshold for which the amount of energy absorbed offers positive feedback, leading to a large increase in the amount of energy absorbed [82]. For the same pulse energy, the shorter pulses have a high intensity overcoming losses due to the three body recombination, de-excitation, photon recombination, Bremsstrahlung emission and plasma expansion $[82,83]$ and reach the ignition threshold. However, longer pulses requires more pulse energy to reach the same intensity and overcome the ignition threshold. The model for the plasma absorption implicitly considers that the plasma absorption occurs at the same time as the start of the ablation. The experimental results presented in Figure 7 show that it is not the case for the present experimental setup as the linear coupling between the fluence and the amount of ablated material stops around $50 \mathrm{~J} . \mathrm{cm}^{-1}$. Furthermore, previous modelling work has found similar behaviour for the plasma ignition [84]. Thus, it is likely that a better description of the plasma will improve the prediction of the model for a fluence over $50 \mathrm{~J} . c m^{-2}$.

Finally, the good agreement between the experimental results and the model for boron doped diamond validates the methodology for the prediction of the thermal stability of diamond during pulsed laser ablation. In the following section, the same methodology is applied for tetrahedral amorphous carbon thus providing further information about the thermal stability for diamond-like carbon thin films that cannot be directly obtained $[5,6]$.

\subsection{Tetrahedral amorphous carbon}

As mentioned previously, the value of the reflectivity for graphite at $248 \mathrm{~nm}$ is not precisely known as a function of the temperature. However, previous pump and probe experiments show that the reflectivity decreases at higher temperature [73]. The error in the model prediction due to the uncertainty of the reflectivity value cannot be estimated analytically and therefore, several values for the reflectivity have been tested numerically. For this investigation, plasma absorption is not taken into account to simplify the discussion. The free parameters used in the implementation of the plasma model are calculated using the experimental results, see (5), which could counterbalance the influence of the reflectivity on the amount of energy reaching the target (by changing the free parameter values) and biasing the discussion about the reflectivity. The position of the interfaces for four cases is shown in Figure 8. It is clear that the reflectivity has a significant influence on the position of the interfaces (graphite/diamond and graphite/vacuum) over the whole range of fluence. For fluence lower than $2.5 \mathrm{~J} . \mathrm{cm}^{-2}$ (fluence for the maximum swelling), the influence of the reflectivity is not strong; an error of $50 \%$ in the value of the reflectivity only introduces around $50 \mathrm{~nm}$ error in the position of the interface between the graphite and vacuum and around $100 \mathrm{~nm}$ for the position of the interface between graphite and diamond. However, for fluence over $10 \mathrm{~J} . \mathrm{cm}^{-2}$, the error increases 


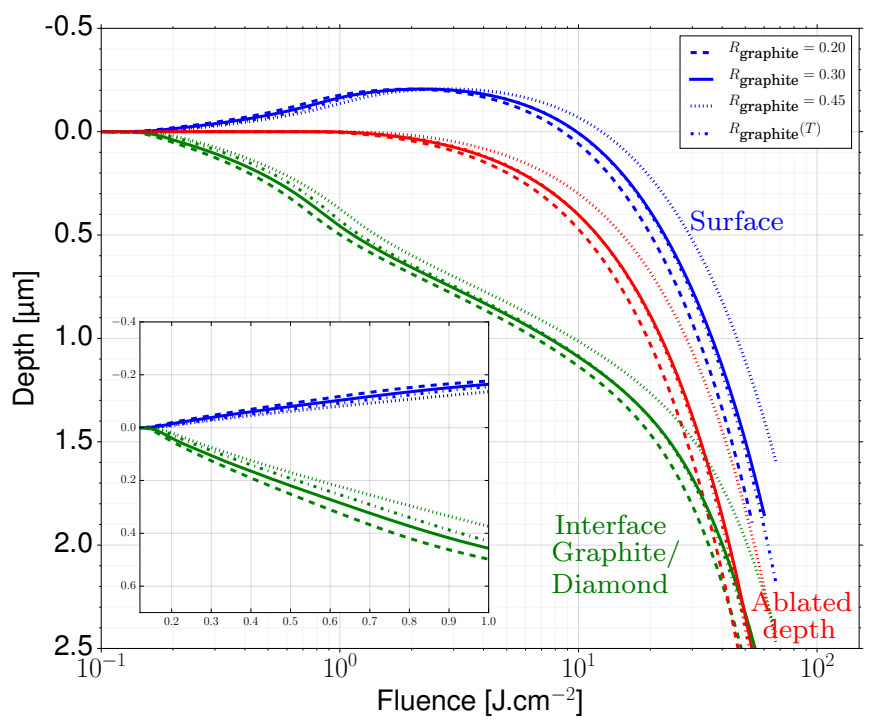

Figure 8: Position of the interfaces and the amount of ablated material for different value of the reflectivity for the graphite phase and for the function given in Table 3. The red, blue and green lines are the ablated depth, the interface position of graphite/vacuum and interface position of diamond/graphite respectively.

proportionally with the fluence. Several points explain these results. For low fluence, a large part of the energy is absorbed when ta- $\mathrm{C}$ is the top material, so changes in the reflectivity of the graphite phase will not change the amount of energy absorbed. As the fluence increases, the start of graphitisation occurs earlier, which means that a large part of the pulse is absorbed when graphite is the surface material. For fluence over $10 \mathrm{~J} . c m^{-2}$, most of the energy is absorbed when the graphite is the surface material, which leads to a large variation in the position of the interfaces, see Figure 8.

The value of the graphite reflectivity has a notable influence on the value of the position of the interfaces especially for high fluence. However, it does not change the trend presented by the numerical experiments. The graphitisation starts at $0.2{\mathrm{~J} . \mathrm{cm}^{-3}}^{-3}$ and increases until the

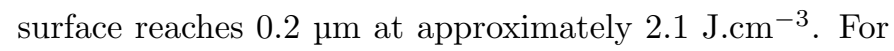
higher fluence, ablation overcomes the swelling and the surface recedes. However, the model results for high fluence cannot be used to estimate the variation of the reflec-

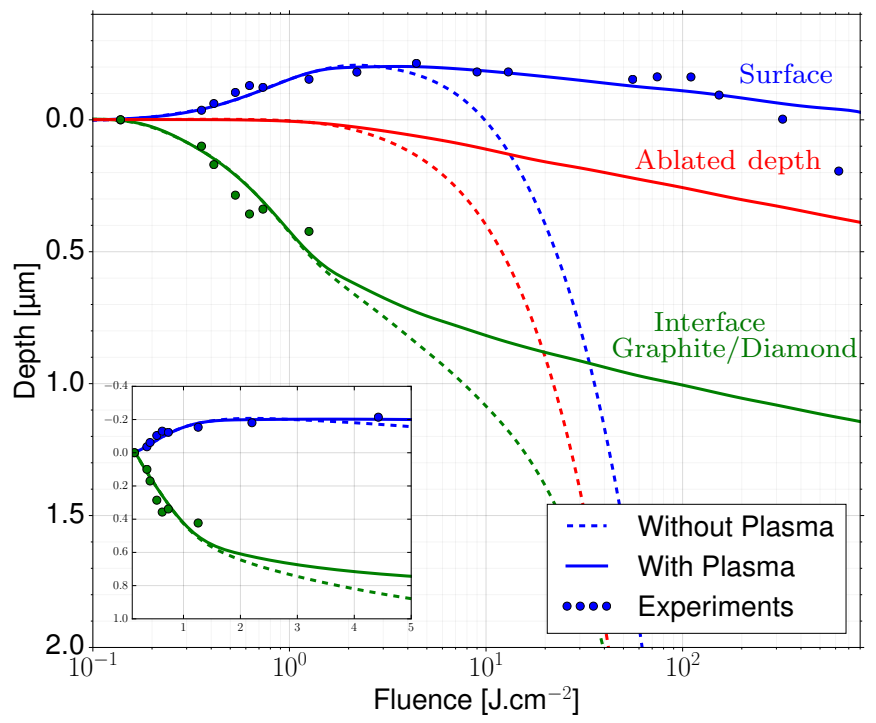

Figure 9: Blue circles are the experimental data from [33]. Green circles are the position of the graphite-ta-C interface estimated using the relation (1). The red, blue and green lines are the ablated depth, the interface position of graphite/vacuum and interface position of diamond/graphite respectively.

tivity due to the ignition of the plasma, which decreases the amount of absorbed energy. It must be noted that the numerical results using these four reflectivity values are all in fairly good agreement with the available numerical results for low fluence. Thus, it does not give us enough information to distinguish between the different cases. In the following simulations, the reflectivity is dependent on the temperature (see Table 3) [73], which leads to better agreement with the experiments.

The experimental and numerical position of the surface for ta-C ablated as well as the numerical results for the position of the interface between diamond and graphite and the amount of ablated material by $20 \mathrm{~ns}$ pulsed laser at $248 \mathrm{~nm}$ are presented in Figure 9. It must be noted that the experimental position of the interfaces between graphite and diamond, presented in Figure 9, has been calculated using the mass conservation, Equation (1), until

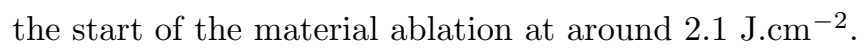

Without considering the plasma shielding, the model is in excellent agreement with the experiments for fluence lower than $3 \mathrm{~J} . \mathrm{cm}^{-3}$. The model is used to simulate the 


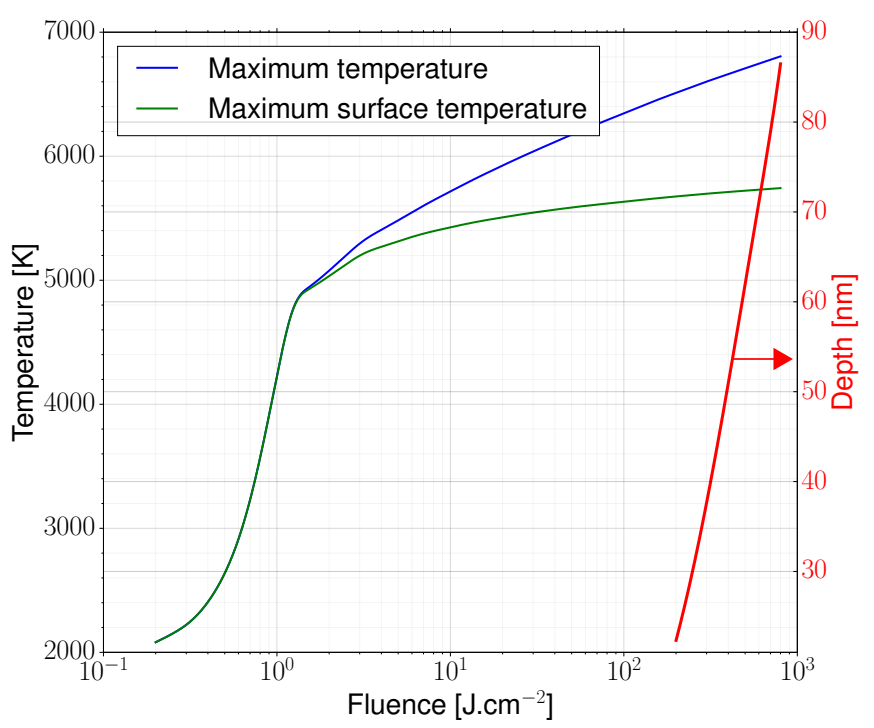

Figure 10: Maximum surface temperature and maximum temperature during the simulation of the interaction between the laser and ta- $\mathrm{C}$ as a function of fluence. The red solid line represents the maximum depth such as the temperature is equal to $6500 \mathrm{~K}$.

interaction between the diamond and the graphite during pulsed laser ablation. Therefore, it is particularly interesting that the predictions are accurate for low fluence and shows that the modelling framework chosen can correctly predict the surface graphitisation of diamond during pulsed laser ablation. Using the simple description of the plasma absorption, (5), it is possible to estimate the amount of energy absorbed by the plasma and extend the range of validity of the model. The parameters $a$ and $b$ have been evaluated using the experimental data and are respectively equal to $5 \times 10^{8} \mathrm{~m}^{-1}$ and $0 \mathrm{~J}^{-1} \cdot \mathrm{cm}^{2}$. These values are consistent with the literature for the ablation of graphite previously done with laser pulses at $532 \mathrm{~nm}$ with a FWHM of $7 \mathrm{~ns}$ [85] and at $1064 \mathrm{~nm}$ with a FWHM of $13 \mathrm{~ns}$ [34]. The value of $a$ increases with a decrease of the wavelength $\left(3 \times 10^{5} \mathrm{~m}^{-1}\right.$ at $1064 \mathrm{~nm}$ and $2 \times 10^{6} \mathrm{~m}^{-1}$ at $532 \mathrm{~nm}$ ) suggesting that plasma ignition occurs with a lower amount of material ablated. Furthermore, it has been shown that at $532 \mathrm{~nm}$, the free parameter $b$ is equal to zero, suggesting that the plasma absorption is almost independent of the amount of energy inside it. These observations are consistent with the main absorption mecha- nisms of laser pulses at $1064 \mathrm{~nm}$ and $248 \mathrm{~nm}$. For infrared lasers, plasma absorption occurs mainly through inverse Bremsstrahlung with the ion and neutral [82, 83], which requires a large electron density and temperature to ignite, so that the parameter $b$ should not be zero. In contrast. for UV lasers, plasma absorption is dominated by photoionisation processes that does not require a large electron population generated by an increase of temperature $[86,83]$ so the parameter $b$ should be small. It must be noted that the variation of the parameter $a$ is also consistent with the absorption mechanisms at VIS and UV wavelength. In effect, a decrease of laser wavelength increases the number of atomic electronic levels (especially so that those levels are generally more densely populated) that participate in photo-ionisation, which leads to a step increase in the plasma absorption coefficient [87]. The plasma absorption increases the range for which the model is in good agreement with the experiments and suggests that the inclusion of the plasma in a more detailed manner could provide additional insight into the ablation mechanisms.

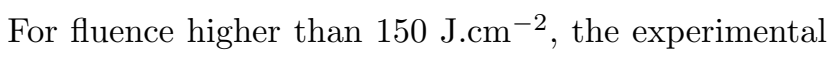
data shows a steep increase in the amount of ablated material that the model presented here cannot explain. This discrepancy for high values of fluence is often found in the literature for pulsed laser ablation modelling [84] and can be explained by explosive boiling/volumetric mass removal, as presented in the work of Demos et al. [88]. The numerical experiments show that the maximum temperature reaches around $6500 \mathrm{~K}$ for fluence over $150 \mathrm{J.cm}{ }^{-2}$. This is close to the widely accepted critical temperature of graphite [74] and is consistent with the beginning of explosive/volumetric mass removal. It has been found that the thickness of material in a thermodynamic state close to the critical is less than $100 \mathrm{~nm}$, see Figure 10. Therefore, the model might not be able to explain the $250 \mathrm{~nm}$ difference between the model and the experiments for a fluence of $622 \mathrm{~J} . \mathrm{cm}^{-2}$. However, it has been found in a previous study that the emission of energy by the 


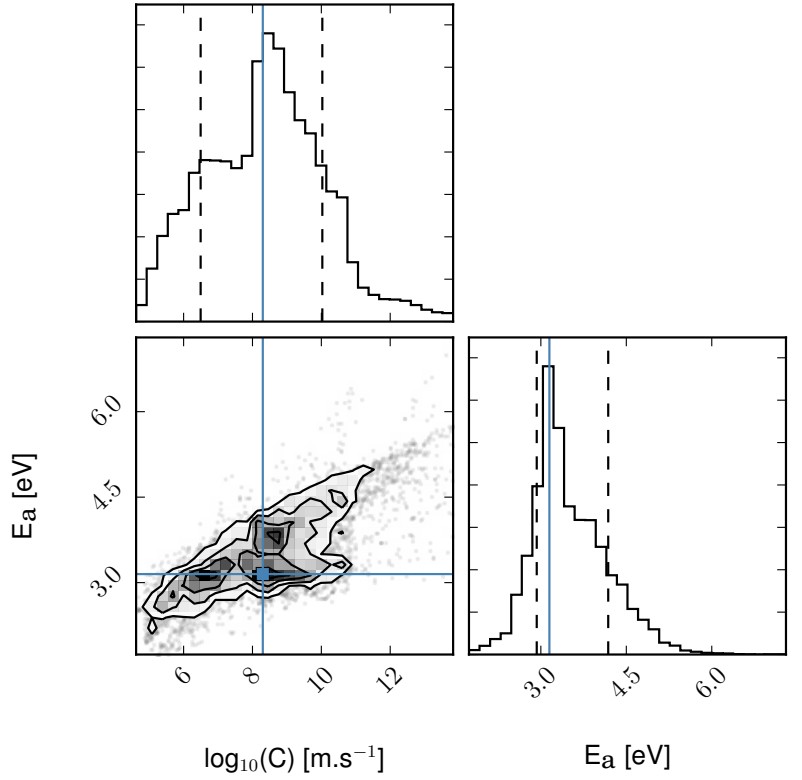

Figure 11: Affine Invariant Markov chain Monte Carlo (MCMC) Ensemble sampler [6] for the the fitting of the surface position predicted from the model to the experimental results using activation energy and the rate of graphitisation as free parameters [89]. The blue line represents the result for the activation energy and the rate of graphitisation from the optimisation using the maximum likelihood method.

heated plasma over the crater due to the bremsstrahlung and photo-recombination processes causes deeper heating without affecting the amount of ablated material [85]. This effect has not been taken into account for this study but it would suggest that the plasma absorption is overestimated. In effect, deeper heat absorption should lead to a larger swelling that should be counterbalanced by an increase of ablation at the surface. It should also lead to a larger layer of material heated over the critical temperature and explain the difference between the experiment and the model for high fluence.

The estimation of the parameters used in Arrhenuis law for the graphitisation of ta-C provides further information about the thermal stability of diamond-like carbon films, see Figure 11. The activation energy for ta-C has previously been determined experimentally at $3.5 \pm 0.9 \mathrm{eV}[4,7]$. The present study provides improved accuracy for the ac- tivation energy $\left(3.15_{-0.22}^{+1.0} \mathrm{eV}\right)$ and also provides an estimation for the rate of graphitisation $\left(\exp \left[\log [10] \times 8.3_{-1.8}^{+1.72}\right]\right.$ m.s ${ }^{-1}$ ), see Figure 11.

\section{Conclusion}

Theoretical and experimental results have been used to investigate the ablation of diamond and related materials with nanosecond laser pulses. The dependence of the ablation rate on laser fluence has been analysed for a wide range of values of laser fluence and two dissimilar types of diamond and related material. The experimental data shows a swelling of the surface for low fluence and an ablation of the surface for high fluence, see Figure 5.

Furthermore, the behaviour of the diamond during laser ablation has been addressed theoretically within a one dimensional modelling framework. The model takes into account the transition between graphite and diamond/taC, the jump of density, the plasma absorption and the evaporation of the material during nanosecond pulse ablation. By solving the system of equations numerically, the space-time distribution of temperature is obtained from the model within the diamond/graphite target. The positions of the interfaces are also obtained from the model. The model shows excellent agreement with the experimental data until the onset of the explosive/volumetric mass removal for ta-C and plasma absorption for boron doped diamond. Model presented in this study is the first one to address the particularity (phase change with change of density) of the interaction between the diamond and related materials with a pulsed laser beam. In effect, previous studies only reported the thickness and the position of the surface without providing a complete modelling framework taking into account the transition of the optical and thermal properties and the dynamic position of the interfaces (diamond/graphite and graphite/vacuum). Interestingly, the model provides insights into the ablation process. It shows that using the quasi-stationary annealing threshold for the graphitisation of diamond and related material 
does not give accurate results. This work shows that the Arrhenuis law provides accurate predictions for the rate of graphitisation during pulsed laser ablation for two allotropes of carbon with a large amount of $\mathrm{sp}^{3}$ bounds. The present study provides a new method to obtain the activation energy and the graphitisation rate for diamond-like carbon, and provides numerical estimations for a ta-C with around $80 \% \mathrm{sp}^{3}$ bounds with an improved accuracy.

In recent years, the interaction between the material ablated from the surface and the laser has been investigated in detail, which demonstrates the importance of accurately modelling the temperature dependence of key physical properties, [29, 48, 90], an aspect that has been strongly emphasised in this paper. The behaviour of the subsurface during ablation has not been heavily studied due to the difficulty of measuring the microstructure inside the material and relating it to the processes that created it. Diamond ablation presents an interesting case for which the maximum position for the graphitisation temperature can be measured after the removal of the graphite layer (using acidic solutions [25] or a furnace in an oxiding atmosphere [33]) putting further constraint on the results of the model. Future studies should provide additional information about the phenomena occurring inside the material during pulsed laser ablation.

\section{Acknowledgements}

This work is supported by the STEEP, Marie Curie Initial Training Network supported by the European Commission with the seventh framework program under the grant agreement $n^{\circ} 316560$.

\section{References}

[1] C. Z. Wang, K. M. Ho, M. D. Shirk, and P. A. Molian, "LaserInduced Graphitization on a Diamond ( 111 ) Surface," Phys. Rev. Lett., vol. 85, no. 19, pp. 4092-4095, 2000.

[2] F. S. Phinney, "Graphitization of Diamond.," Science, vol. 120, pp. 393-4, sep 1954.
[3] G. Davies and T. Evans, "Graphitization of dimaond at zero pressure and at a high pressure," Proc. R. Soc. London, vol. 328, pp. 413-427, 1972.

[4] D. S. Grierson, A. V. Sumant, A. R. Konicek, T. A. Friedmann, J. P. Sullivan, R. W. Carpick, D. S. Grierson, A. V. Sumant, A. R. Konicek, T. A. Friedmann, and J. P. Sullivan, "Thermal stability and rehybridization of carbon bonding in tetrahedral amorphous carbon Thermal stability and rehybridization of carbon bonding in tetrahedral," vol. 033523, 2010.

[5] A. C. Ferrari, S. E. Rodil, J. Robertson, and W. I. Milne, "Is stress necessary to stabilise sp3 bonding in diamond-like carbon?," Diam. Relat. Mater., vol. 11, no. 3-6, pp. 994-999, 2002.

[6] D. W. Hogg, J. Bovy, and D. Lang, "Data analysis recipes: Fitting a model to data," eprint arXiv, vol. 1008, p. 4686, 2010.

[7] A. Ferrari and J. Robertson, "Interpretation of Raman spectra of disordered and amorphous carbon," Phys. Rev. B, vol. 61, no. 20, pp. 14095-14107, 2000.

[8] A. Tokmakoff, W. Banholzer, and M. Fayer, "Thermal diffusivity measurements of natural and isotopically enriched diamond by picosecond infrared transient grating experiments," Appl. Phys. A, vol. 90, pp. 87-90, 1993.

[9] J. E. Field, The Properties of Natural and Synthetic Diamond, vol. 28. Academic Press, London, 1993.

[10] R. P. Mildren, "Intrinsic Optical properties of Diamond," in Opt. Eng. Diam., vol. 1, ch. First, pp. 1-34, Weinheim, Germany: John Wiley \& Sons, jan 2013.

[11] C. Donnet and A. Erdemir, Tribology of Diamond-like Carbon Films: Fundamentals and Applications. Springer US, 2007.

[12] C. Everson and P. Molian, "Fabrication of polycrystalline diamond microtool using a Q-switched Nd:YAG laser," Int. J. Adv. Manuf. Technol., vol. 45, pp. 521-530, mar 2009.

[13] T. Kononenko, V. Ralchenko, A. Bolshakov, V. Konov, P. Allegrini, M. Pacilli, G. Conte, and E. Spiriti, "All-carbon detector with buried graphite pillars in CVD diamond," Appl. Phys. A, vol. 114, pp. 297-300, nov 2014.

[14] M. Girolami, P. Allegrini, G. Conte, D. M. Trucchi, V. G. Ralchenko, and S. Salvatori, "Diamond Detectors for UV and X-Ray Source Imaging," IEEE Electron Device Lett., vol. 33, no. 2, pp. 224-226, 2012.

[15] R. S. Sussmann, CVD Diamond for Electronic Devices and Sensors. Wiley Series in Materials for Electronic \& Optoelectronic Applications, Chichester, West Sussex, United Kingdom: John Wiley \& Sons, first ed., 2009.

[16] J. V. Beck, A. Osman, and G. Lu, "Maximum temperatures in Diamond Heat Spreaders Using the Surface Element Method," ASME J. Heat Transf., vol. 115, no. 1, pp. 51-57, 1993.

[17] H. K. Mao and R. J. Hemley, "Optical transitions in diamond at ultrahigh pressures," Lett. to Nat., vol. 351, pp. 721-724, 1991. 
[18] M. Pacella, P. Butler-Smith, D. A. Axinte, and M. Fay, "FIB/TEM/EELS micro/nanometric investigations of the effects of laser ablation on the diamond/binder structure in polycrystalline diamond composites," J. Mater. Process. Technol., vol. 214, pp. 1153-1161, may 2014.

[19] G. Zhang, B. Zhang, Z. Deng, and J. Chen, "An Experimental Study on Laser Cutting Mechanisms of Polycrystalline Diamond Compacts," CIRP Ann. - Manuf. Technol., vol. 56, no. 1, pp. 201-204, 2007.

[20] M. D. Shirk, P. A. Molian, and A. P. Malshe, "Ultrashort pulsed laser ablation of diamond," J. Laser Appl., vol. 10, no. 2, p. 64, 1998.

[21] N. Dahotre and S. Harimkar, Laser fabrication and machining of materials, vol. 1. Springer, 2008.

[22] S. Garnov, V. Konov, and T. Kononenko, "Microsecond Laser Material Processing at $1.06 \mu \mathrm{m}, "$ LASER Phys. - Rapid Commun., vol. 14, no. 6, pp. 910-915, 2004.

[23] J. Jandeleit, A. Horn, E.-W. Kreutz, and R. Poprawe, "Micromachining of metals and ceramics by nano- and picosecond laser radiation," 1997.

[24] C. Momma, U. Knop, and S. Nolte, "Laser cutting of slotted tube coronary stents - state-of-the-art and future developments," Prog. Biomed. Res., vol. 1, no. February, pp. 39-44, 1999.

[25] T. Kononenko, V. Kononenko, S. Pimenov, E. Zavedeev, V. Konov, V. Romano, and G. Dumitru, "Effects of pulse duration in laser processing of diamond-like carbon films," Diam. Relat. Mater., vol. 14, pp. 1368-1376, aug 2005.

[26] F. Dausinger, H. Hugel, and V. I. Konov, "Micromachining with ultrashort laser pulses: from basic understanding to technical applications," 2003.

[27] M. R. H. Knowles, G. Rutterford, D. Karnakis, and A. Ferguson, "Micro-machining of metals, ceramics and polymers using nanosecond lasers," Int. J. Adv. Manuf. Technol., vol. 33, no. 12, pp. 95-102, 2007.

[28] G. B. J. Cadot, D. A. Axinte, and J. Billingham, "Continuous trench; pulsed laser ablation for micro-machining applications," Int. J. Mach. Tools Manuf., vol. 107, pp. 1-36, 2016.

[29] D. Autrique, G. Clair, D. L'Hermite, V. Alexiades, A. Bogaerts, and B. Rethfeld, "The role of mass removal mechanisms in the onset of ns-laser induced plasma formation," J. Appl. Phys., vol. 114, no. 2, 2013.

[30] H. Ohfuji, T. Okuchi, S. Odake, H. Kagi, H. Sumiya, and T. Irifune, "Micro-/nanostructural investigation of laser-cut surfaces of single- and polycrystalline diamonds," Diam. Relat. Mater., vol. 19, pp. 1040-1051, jul 2010.

[31] S. Odake, H. Ohfuji, T. Okuchi, H. Kagi, H. Sumiya, and T. Irifune, "Pulsed laser processing of nano-polycrystalline diamond:
A comparative study with single crystal diamond," Diam. Relat. Mater., vol. 18, pp. 877-880, may 2009.

[32] M. Seal, "Graphitization of diamond," Nature, vol. 185, pp. 522-523, 1960.

[33] V. I. Konov, T. V. Kononenko, and V. V. Kononenko, "Laser Micro- and Nanoprocessing of Diamond Materials," in Opt. Eng. Diam. (M. Rich, ed.), ch. 12, pp. 385-443, Weinheim, Germany: John Wiley \& Sons, jan 2013.

[34] N. M. Bulgakova and A. V. Bulgakov, "Pulsed laser ablation of solids : transition from normal vaporization," Appl. Phys. A, vol. 208, no. 2, pp. 199-208, 2001.

[35] R. K. Singh, O. W. Holland, and J. Narayan, "Theoretical model for deposition of superconducting thin films using pulsed laser evaporation technique," J. Appl. Phys., vol. 68, no. 1, p. 233, 1990.

[36] N. A. Vasantgadkar, U. V. Bhandarkar, and S. S. Joshi, "A finite element model to predict the ablation depth in pulsed laser ablation," Thin Solid Films, vol. 519, pp. 1421-1430, dec 2010.

[37] Q. Lu, S. S. Mao, X. Mao, and R. E. Russo, "Delayed phase explosion during high-power nanosecond laser ablation of silicon," Appl. Phys. Lett., vol. 80, no. 17, p. 3072, 2002.

[38] D. Marla, U. V. Bhandarkar, and S. S. Joshi, "Transient Analysis of Laser Ablation Process With Plasma Shielding: OneDimensional Model Using Finite Volume Method," J. Micro Nano-Manufacturing, vol. 1, p. 011007, mar 2013.

[39] A. H. A. Lutey, "An improved model for nanosecond pulsed laser ablation of metals," J. Appl. Phys., vol. 114, no. 8, p. $083108,2013$.

[40] A. Bulgakov and N. Bulgakova, "Thermal model of pulsed laser ablation under the conditions of formation and heating of a radiation-absorbing plasma," Quantum Electron., vol. 433, no. 29 , pp. 433 - $437,1999$.

[41] G. Lamé and B. P. Clapeyron, "Mémoire sur la solidification par refroidissement d'un globe liquide," Ann. Chim. Phys., vol. 47, pp. 250-256, 1831.

[42] I. Langmuir, "The Vapor Pressure of Metallic Tungsten," Phys. Rev., vol. 2, no. 5, pp. 329-342, 1913.

[43] L. H. Long, L. Brewer, P. W. Gilles, and F. A. Jenkins, "The Vapor Pressure and Heat of Sublimation of Graphite," J. Chem. Phys., vol. 16, no. 8, p. 797, 1948.

[44] A. Morozov, "Thermal model of pulsed laser ablation: back flux contribution," Appl. Phys. A, vol. 79, pp. 997-999, jul 2004.

[45] H. Leider, O. Krikorian, and D. Young, "Thermodynamic properties of carbon up to the critical point," Carbon N. Y., vol. 11, pp. 555-563, oct 1973.

[46] V. R. Voller, "An overview of numerical methods for solving phase change problems," in Adv. Numer. Heat Transf., 1996. 
[47] J. W. Demmel, S. C. Eisenstat, J. R. Gilbert, X. S. Li, and J. W. H. Liu, "A supernodal approach to sparse partial pivoting," SIAM J. Matrix Anal. Appl., vol. 20, no. 3, pp. 720-755, 1999.

[48] D. Marla, U. V. Bhandarkar, and S. S. Joshi, "Models for predicting temperature dependence of material properties of aluminum," J. Phys. D. Appl. Phys., vol. 47, p. 105306, mar 2014.

[49] Element Six ${ }^{\top M}$, "Thermal and Optical Properties of Element Six materials," 2016.

[50] B. Vrolijk, "Optical Grade CVD, Single Crystal Diamond," Elem. Six ${ }^{\text {TM }}$, p. 2, 2014.

[51] D. Wu, Z. L. Wang, Q. Luo, C. Z. Gu, N. L. Wang, C. Y. Li, X. Y. Lu, and Z. S. Jin, "Optical properties of boron-doped diamond," Phys. Rev. B, vol. 73, pp. 1-5, 2006.

[52] F. D. Rossini and R. S. Jessup, "Heat and free energy of formation of carbon dioxide, and of the transition between graphite and diamond," J. Res. Natl. Bur. Stand. (1934)., vol. 21, no. 4, p. $491,1938$.

[53] T. Inushima, A. Ogasawara, T. Shiraishi, S. Ohya, S. Karasawa, and H. Shiomi, "Optical and electrical investigation of borondoped homoepitaxial diamond," Diam. Relat. Mater., vol. 6, no. 5-7, pp. 835-838, 1997.

[54] E. Gheeraert, A. Deneuville, and J. Mambou, "Boron-related infra-red absorption in homoepitaxial diamond films," Diam. Relat. Mater., vol. 7, no. 10, pp. 1509-1512, 1998.

[55] V. A. Krivchenko, D. V. Lopaev, P. V. Minakov, V. G. Pirogov, a. T. Rakhimov, and N. V. Suetin, "Study of polycrystalline boron-doped diamond films by Raman spectroscopy and optical absorption spectroscopy," Tech. Phys., vol. 52, no. 11, pp. 14711474, 2007.

[56] M. Ficek, M. Sobaszek, M. Gnyba, J. Ryl, Ł. Gołuński, M. Smietana, J. Jasiński, P. Caban, and R. Bogdanowicz, "Optical and electrical properties of boron doped diamond thin conductive films deposited on fused silica glass substrates," Appl. Surf. Sci., vol. 387, pp. 846-856, 2016.

[57] D. Prikhodko, S. Tarelkin, V. Bormashov, A. Golovanov, M. Kuznetsov, D. Teteruk, A. Volkov, and S. Buga, "Thermal conductivity of synthetic boron-doped single-crystal HPHT diamond from 20 to $400 \mathrm{~K}$," MRS Commun., pp. 1-6, 2016.

[58] S. A. Herr, J. V. Beck, J. J. McGrath, S. Sahli, and M. Aslam, "An optimized experimental method for measuring thermal conductivity of thin, boron-doped diamond films," Rev. Sci. Instrum., vol. 66, no. 10, p. 4967, 1995.

[59] C. Moelle, M. Werner, F. Sz ucs, D. Wittorf, M. Sellschopp, J. von Borany, H.-J. Fecht, and C. Johnston, "Specific heat of single-, poly-and nanocrystalline diamond," Diam. Relat. Mater., vol. 7, pp. 499-503, 1998.

[60] E. A. Ekimov, V. Ralchenko, and A. Popovich, "Synthesis of su- perconducting boron-doped diamond compacts with high elastic moduli and thermal stability," Diam. Relat. Mater., vol. 50, pp. 15-19, 2014.

[61] E. V. Ivakin, A. V. Sukhodolov, V. G. Ralchenko, A. V. Vlasov, and A. V. Khomich, "Measurement of thermal conductivity of polycrystalline CVD diamond by laser-induced transient grating technique," Quantum Electron., vol. 32, no. 4, pp. 367-372, 2007.

[62] B. Wu and Y. C. Shin, "Absorption coefficient of aluminum near the critical point and the consequences on high-power nanosecond laser ablation," Appl. Phys. Lett., vol. 89, no. 11, p. 111902, 2006.

[63] A. Libassi, A. C. Ferrari, V. Stolojan, B. K. Tanner, J. Robertson, and L. M. Brown, "Density, sp3 content and internal layering of DLC films by X-ray reflectivity and electron energy loss spectroscopy," Diam. Relat. Mater., vol. 9, no. 3, pp. 771-776, 2000.

[64] H. Schulz, H. J. Scheibe, P. Siemroth, and B. Schultrich, "Pulsed arc deposition of super-hard amorphous carbon films," Appl. Phys. A Mater. Sci. Process., vol. 78, no. 5, pp. 675-679, 2004.

[65] J. I. Larruquert, L. V. Rodriguez-de Marcos, J. A. Mendez, P. J. Martin, and A. Bendavid, "High reflectance ta-C coatings in the extreme ultraviolet," Opt. Express, vol. 21, no. 23, pp. 435-438, 2013.

[66] M. Hakovirta, J. E. Vuorinen, X. M. He, M. Nastasi, and R. B. Schwarz, "Heat capacity of hydrogenated diamond-like carbon films," Appl. Phys. Lett., vol. 77, no. 15, p. 2340, 2000.

[67] M. Shamsa, W. L. Liu, A. A. Balandin, C. Casiraghi, W. I. Milne, and A. C. Ferrari, "Thermal conductivity of diamondlike carbon films," Appl. Phys. Lett., vol. 89, no. 16, pp. 1-3, 2006.

[68] S. R. P. Silva, Properties of Amorphous Carbon. EMIS Datareviews Series n.29, Herts, UK: INSPEC, 1st ed., 2003.

[69] B. Tay, X. Shi, L. Cheah, and D. Flynn, "Optical properties of tetrahedral amorphous carbon films determined by spectroscopic ellipsometry," Thin Solid Films, vol. 308-309, pp. 268$272,1997$.

[70] Z. Di, A. Huang, R. K. Y. Fu, P. K. Chu, L. Shao, T. Höchbauer, M. Nastasi, M. Zhang, W. Liu, Q. Shen, S. Luo, Z. Song, and C. Lin, "Thermal stability of diamondlike carbon buried layer fabricated by plasma immersion ion implantation and deposition in silicon on insulator," J. Appl. Phys., vol. 98, no. 5, pp. 15, 2005.

[71] A. I. Savvatimskiy, "Liquid carbon density and resistivity.," J. Phys. Condens. Matter, vol. 20, p. 114112, mar 2008.

[72] A. B. Djurišić and E. H. Li, "Optical properties of graphite," J. Appl. Phys., vol. 85, no. 10, p. 7404, 1999. 
[73] A. M. Malvezzi and N. Bloembergen, "Time-Resolved Picosecond Optical Measurements of Laser-Excited Graphite," Phys. Rev. Lett., vol. 57, no. 1, pp. 146-149, 1986.

[74] A. Savvatimskiy, "Measurements of the melting point of graphite and the properties of liquid carbon (a review for 19632003)," Carbon N. Y., vol. 43, pp. 1115-1142, may 2005.

[75] M. Akoshima, B. Hay, J. Zhang, L. Chapman, and T. Baba, "International Comparison on Thermal-Diffusivity Measurements for Iron and Isotropic Graphite Using the Laser Flash Method in CCT-WG9," Int. J. Thermophys., vol. 34, pp. 763-777, jun 2012.

[76] P. W. Butler-smith, D. A. Axinte, M. Pacella, and M. W. Fay, "Micro / nanometric investigations of the effects of laser ablation in the generation of micro-tools from solid CVD diamond structures," J. Mater. Process. Technol., vol. 213, pp. 194-200, 2013.

[77] G. Pottlacher, R. Hixson, S. Melnitzky, E. Kaschnitz, M. Winkler, and H. Jäger, "Thermophysical properties of POCO AXF5Q graphite up to melting," Thermochim. Acta, vol. 218, pp. 183-193, may 1993.

[78] V. N. Korobenko, A. I. Savvatimski, and R. Cheret, "Graphite Melting and Properties of Liquid Carbon," Int. J. Thermophys., vol. 20, no. 4, pp. 1247-1256, 1999.

[79] A. M. K. Fujii, Effect of Nanoporosity on the Thermal Conductivity of Amorphous Carbon. PhD thesis, University of California, 2014.

[80] M. Komlenok, S. Pimenov, V. V. Kononenko, V. I. Konov, and H.-J. Scheibe, "Laser Microstructuring of the surface of ta-C films," J. Nano Microsyst. Tech., vol. 3, pp. 48-53, 2008.

[81] N. Dubrovinskaia, R. Wirth, J. Wosnitza, T. Papageorgiou, H. F. Braun, N. Miyajima, and L. Dubrovinsky, "An insight into what superconducts in polycrystalline boron-doped diamonds based on investigations of microstructure.," Proc. Natl. Acad. Sci. U. S. A., vol. 105, no. 33, pp. 11619-11622, 2008.

[82] V. Morel and A. Bultel, "Theoretical study of the formation mechanism of laser-induced aluminum plasmas using Nd:YAG fundamental, second or third harmonics," Spectrochim. Acta Part B At. Spectrosc., vol. 94-95, pp. 63-70, 2014.

[83] D. Autrique, I. Gornushkin, V. Alexiades, Z. Chen, A. Bogaerts, and B. Rethfeld, "Revisiting the interplay between ablation, collisional, and radiative processes during ns-laser ablation," Appl. Phys. Lett., vol. 103, no. 17, 2013.

[84] M. Stafe, "Theoretical photo-thermo-hydrodynamic approach to the laser ablation of metals," J. Appl. Phys., vol. 112, no. 12, p. 123112, 2012.

[85] N. M. Bulgakova, A. B. Evtushenko, Y. G. Shukhov, S. I. Kudryashov, and A. V. Bulgakov, "Role of laser-induced plasma in ultradeep drilling of materials by nanosecond laser pulses,"
Appl. Surf. Sci., vol. 257, pp. 10876-10882, oct 2011.

[86] R. Rozman, I. Grabec, and E. Govekar, "Influence of absorption mechanisms on laser-induced plasma plume," Appl. Surf. Sci., vol. 254, pp. 3295-3305, mar 2008.

[87] V. Morel, A. Bultel, and B. G. Chéron, "Modeling of thermal and chemical non-equilibrium in a laser-induced aluminum plasma by means of a Collisional-Radiative model," Spectrochim. Acta - Part B At. Spectrosc., vol. 65, no. 9-10, pp. 830$841,2010$.

[88] S. G. Demos, R. A. Negres, R. N. Raman, Michael D. Feit, K. R. Manes, and A. M. Rubenchik, "Relaxation dynamics of nanosecond laser superheated material in dielectrics," Optica, vol. 2, no. 8, pp. 765-772, 2015.

[89] D. Foreman-Mackey, "corner.py: Scatterplot matrices in Python," J. Open Source Softw., vol. 1, no. 2, pp. 1-2, 2016.

[90] G. Galasso, M. Kaltenbacher, A. Tomaselli, and D. Scarpa, "A unified model to determine the energy partitioning between target and plasma in nanosecond laser ablation of silicon," J. Appl. Phys., vol. 117, no. 12, p. 123101, 2015. 